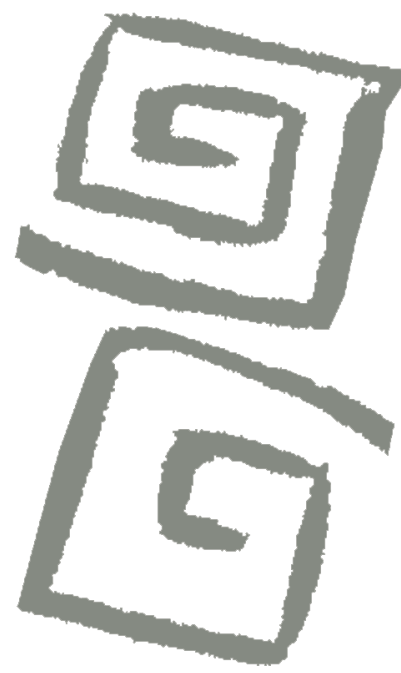

\title{
Análisis filosófico sobre la concepción neurocientífica de los rasgos autistas: aportes desde el normativismo vital de Georges Canguilhem
}

\author{
A philosophical analysis of the neuroscientific \\ conception of autistic traits: contributions from Georges \\ Canguilhem's biological normativity
}

Clara Castañares ${ }^{1}$, Luis Bagatolli², Guillermo Folguera ${ }^{3}$

'Bioquímica. Doctorando en Neurociencias. Becaria, Consejo Nacional de Investigaciones Científicas y Técnicas, Instituto Ferreyra Instituto de Investigación Médica Mercedes y Martín Ferreyra, Universidad Nacional de Córdoba: Córdoba, Argentina. $\square$

${ }^{2}$ Doctor en Química. Investigador principal, Consejo Nacional de Investigaciones Científicas y Técnicas. Director interino, Instituto Ferreyra Instituto de Investigación Médica Mercedes y Martín Ferreyra Universidad Nacional de Córdoba; Córdoba, Argentina. $\triangle$ iD

${ }^{3}$ Biólogo. Filósofo. Investigador independiente, Consejo Nacional de Investigaciones Científicas y Técnicas. Profesor, Universidad de Buenos Aires; Ciudad Autónoma de Buenos Aires, Argentina. $\square$ (iD)
RESUMEN A partir de la obra Lo normal y lo patológico de Georges Canguilhem, publicada en 1943, se explora cómo las neurociencias definen y legitiman, en la actualidad, la existencia de los rasgos autistas como expresión subclínica de autismo. La hipótesis general del presente trabajo es que en la bibliografía especializada de este ámbito parecen consolidarse diferentes supuestos asumidos en el marco de una perspectiva naturalista de la salud. Tales supuestos refieren a que los comportamientos deben ser explicados estrictamente en términos biológicos, que existe un parámetro de normalidad de carácter objetivo y estadístico, y que las conductas de los individuos pueden ser analizadas separadamente del contexto. Retomando algunos aspectos de la propuesta de Canguilhem sobre salud, normalidad y normatividad, se analiza de qué manera las neurociencias otorgan una cualidad cuasi-patológica a una noción descriptiva como la de rasgos autistas. A nuestro entender, este proceso involucra la asunción apriorística y acrítica de supuestos que impiden poner en discusión aspectos elementales asociados a la naturaleza del autismo y que conducen a patologizar la diferencia entre personas.

PALABRAS CLAVES Neurociencias; Trastorno del Espectro Autista; Filosofía; Medicalización.

\begin{abstract}
Taking Georges Canguilhem's 1943 book The normal and the pathological as a starting point, this article explores the ways in which the neurosciences define, validate, and legitimize the existence of autistic traits as a subclinical expression of autism. The general hypothesis is that different assumptions based on a naturalistic perspective of health and disease have become consolidated in the specialized literature. Such assumptions include that behaviors should be explained strictly in biological terms, that there is an objective and statistical parameter of normality, and that individuals' behaviors can be analyzed independently of their context. Based on some aspects of Canguilhem's arguments regarding health, normality, and normativity, we analyze the ways in which the neurosciences assign a quasi-pathological quality to the descriptive notion of autistic traits. It is possible to conclude that this process involves certain assumptions taken in an aprioristic and uncritical manner, which inhibits the discussion of key aspects associated with the nature of autism and that end up pathologizing differences between people.
\end{abstract}

KEY WORDS Neurosciences; Autism Spectrum Disorder; Philosophy; Medicalization. 


\section{INTRODUCCIÓN}

Durante las últimas décadas, las neurociencias se han constituido como uno de los campos disciplinares más resonantes de la salud mental. La psiquiatría, considerada como una rama médica que debe aproximarse a los padecimientos con el objetivo último de curarlos $^{(1)}$, ha depositado en ellas la tarea de dilucidar las bases etiológicas que subyacen a las patologías mentales, asumidas como enfermedades del cerebro. Se espera así que los conocimientos acerca de las causas y mecanismos involucrados se traduzcan en intervenciones eficaces para su tratamiento y cura $^{(2,3,4)}$. Dado este lugar central de las neurociencias, es posible reconocer abordajes que se asumen como condiciones necesarias y suficientes para la interpretación y explicación de los comportamientos humanos y diagnósticos psiquiátricos ${ }^{(5,6,7,8)}$; abordajes que nos interesa problematizar para el caso particular del autismo.

En este contexto, cada vez es más frecuente la publicación de artículos del ámbito de las neurociencias que reportan la manifestación de rasgos autistas en diversas poblaciones, diferenciadas por criterios vinculados a la edad, género, demografía, presencia de patologías físicas o mentales previas, entre otros. Para ejemplificar brevemente, hay investigaciones que resaltan la presencia de niveles elevados de rasgos autistas entre personas que han intentado cometer suicidio(9), entre mujeres con anorexia nerviosa ${ }^{(10)}$, entre criminales de una prisión de máxima seguridad $^{(11)}$, etc. La base común de estos trabajos radica en considerar que los rasgos autistas se distribuyen entre la población general ${ }^{(12)}$, lo cual habilita evaluarlos y hallarlos en grupos de personas no autistas, con las más variadas características. Por lo tanto, si los rasgos autistas no son exclusivos de las personas autistas, entonces pueden estar presentes en cualquier individuo siempre que reúna dichas características. Sumado a esto, numerosos autores asimilan la presencia elevada de rasgos autistas a la expresión subclínica de autismo tal como indican Zhao et al.: "estas manifestaciones no clínicas pero significativas de los fenotipos autistas en la población general se conocen como rasgos autistas subclínicos"(13). En este sentido, dos importantes referentes del campo del autismo afirman que "las medidas de los rasgos autistas [...] muestran un continuo fluido entre el autismo diagnosticado y las diferencias individuales subclínicas; [...] parece que, al menos a nivel conductual, uno puede ser 'un poco autista'"(14). Bajo estas premisas, hoy parece haberse instalado la idea de que cualquier individuo es susceptible de ser considerado "un poco autista".

Indagar acerca del modo en que se conceptualiza al autismo, desde un enfoque filosófico, puede ayudarnos a comprender su expansión hacia diversas circunstancias que requieren ser analizadas en su condición de patológicas. Uno de los autores que sin dudas ha realizado aportes significativos en esta dirección es Georges Canguilhem, considerado como uno de los fundadores de la actual teoría francesa del conocimiento. Siendo uno de los filósofos de la ciencia más influyentes, fue además quien dirigió la tesis Folie et déraison de Michel Foucault. Aunque dedicó gran parte de su obra al abordaje histórico-epistemológico de la medicina y la fisiología, no descartó que tales ideas fuesen aplicables a los padecimientos mentales e incluso dedicó algunos párrafos a esta temática. Tal es así que autores contemporáneos han retomado sus postulados para el análisis filosófico de la salud mental en general ${ }^{(4,15,16)}$ y de diagnósticos psiquiátricos en particu$\operatorname{lar}^{(4,17,18,19,20)}$. En este trabajo, nos valdremos de su obra Lo normal y lo patológico, publicada en 1943, para realizar un análisis sobre los aspectos epistemológicos de la distinción de los rasgos autistas como manifestación subclínica de autismo y para indagar acerca de su emergencia, consolidación y expansión en las neurociencias. En su desarrollo, Canguilhem se pregunta mediante qué estrategias la medicina transforma datos estadísticos y descriptivos en ideales biológicos que determinan la normalidad y la salud. Trayendo dicho interrogante a nuestro contexto la pregunta que aquí se aborda es cómo las 
neurociencias otorgan una cualidad (cuasi) patológica a una noción descriptiva como la de los rasgos autistas. De manera general, se reconocen en este proceso tres presupuestos que son asumidos a priori: los comportamientos deben ser explicados fundamentalmente en términos biológicos, existe un parámetro de normalidad de carácter objetivo y estadístico, y las conductas de los individuos pueden ser analizadas independientemente de su contexto.

Este trabajo no pretende ser una revisión bibliográfica exhaustiva y sistemática; en cambio, nos interesa cuestionar los aspectos filosóficos subyacentes a la configuración del autismo que se establecen en las neurociencias a través de una discusión epistemológica. Nuestro objetivo radica en explorar de qué modo es definida, validada y legitimada la existencia de los rasgos autistas como expresión subclínica de autismo. De esta manera, la hipótesis del presente trabajo es que en la bibliografía especializada del ámbito de las neurociencias que hemos examinado, la noción de rasgos autistas como manifestación subclínica de autismo se sostiene y se reproduce mediante la consolidación de diferentes presupuestos que se asumen en el marco de una perspectiva naturalista de la salud y la enfermedad, y que impiden poner en discusión aspectos elementales asociados a la naturaleza del autismo. En este sentido, subyace una fundamentación positivista y biologicista, una desvinculación del sujeto con su contexto y un parámetro de normalidad esencialista y valorativo basado en comportamientos socialmente aceptados.

Con este fin, en la primera sección introduciremos al autismo como objeto de estudio de las neurociencias y como diagnóstico psiquiátrico a la vez que indagaremos acerca del origen de la distinción de los rasgos autistas, procurando comprender el uso que se le da a esta noción y acercando algunas críticas suscitadas al interior del campo en este sentido. En una segunda sección, retomaremos los postulados de Canguilhem para discutir el carácter naturalista de las neurociencias que se ve reflejado sobre los rasgos autistas. Posteriormente, en la tercera sección, repararemos sobre la contracara de los presupuestos que se asumen para validar y reproducir la idea de los rasgos autistas, aportando algunos comentarios al respecto. Finalmente, concluimos que es necesario problematizar estos enfoques acríticos de las neurociencias ya que su aproximación naturalista de la salud mental, fuertemente instalada como única perspectiva posible, conduce a planteos deterministas y esencialistas que derivan en prácticas que patologizan la vida y la diferencia.

\section{AUTISMO Y RASGOS AUTISTAS}

Desde la perspectiva neurocientífica, se afirma que el autismo es un trastorno del neurodesarrollo altamente hereditario, con elevada prevalencia y con una presentación clínica muy heterogénea y variable ${ }^{(21)}$. Su caracterización atravesó numerosas versiones y modalidades desde la primera definición formulada en 1943 por el médico psiquiatra infantil Leo Kanner ${ }^{(22)}$. En la actualidad, el Manual Estadístico de Trastornos Mentales en su quinta versión (DSM-5), lo clasifica dentro de los trastornos del neurodesarrollo y lo denomina específicamente como "trastorno del espectro autista" (TEA). Los criterios diagnósticos involucran síntomas relacionados con déficits en aspectos sociales -interacción y comunicación social- y a una dimensión no social que abarca la presencia de patrones repetitivos o restrictivos de comportamiento y anomalías sensoriales. Si bien se considera que esta condición afecta principalmente a infantes, las personas jóvenes o adultas también pueden recibir este diagnóstico ${ }^{(23)}$.

En relación con los rasgos autistas, $\mathrm{Ni}$ cholas Chown ${ }^{(24)}$ afirma:

La gran mayoría de artículos que hacen referencia al término [rasgos autistas] aparentemente lo hacen bajo el supuesto de que se entenderá sin explicación [...]. Aquellos autores que se refieren específicamente a "rasgos autistas" parecen 
asumir que tales rasgos existen y que esta suposición no es controvertida. ${ }^{(24)}$

En este trabajo, el autor argumenta que la noción de rasgos autistas se ha consolidado como un hecho dado (a fact), a raíz de un proceso de reificación de esta creación conceptual propia del campo del autismo ${ }^{(24)}$. Ciertamente, aunque el uso de esta distinción se ha masificado, pocos investigadores explicitan a qué se refieren con ella y de hecho parecería que no existe una definición precisa. A continuación, intentaremos comprender cuál es el uso que se le da a los rasgos autistas en la bibliografía especializada del ámbito de las neurociencias y posteriormente daremos cuenta de algunas críticas suscitadas al interior de este campo disciplinar.

En términos de Chown, la "creación conceptual" de los rasgos autistas se habría originado en el marco de los primeros estudios sobre autismo con familiares, cuando se observó que algunas personas con un parentesco de primer y segundo grado manifestaban ciertos comportamientos y características similares a las de su familiar diagnosticado con autismo. Este fenómeno se denominó "fenotipo autista ampliado" (BAP, por sus siglas en inglés) y dichas características y comportamientos fueron entendidas como "rasgos autistas"(25). Desde entonces y actualmente, la expresión del BAP y la manifestación de rasgos autistas en personas que no son familiares de individuos diagnosticados con autismo se consideran expresiones subclínicas de autismo ${ }^{(13,25,26)}$. Así lo indican precisamente Zhao et al. al afirmar que "... estas manifestaciones no clínicas pero significativas de los fenotipos autistas en la población general se conocen como rasgos autistas subclínicos"(13). Aunque también hay autores que distinguen los rasgos autistas como un fenómeno independiente del autismo, como sugiere el trabajo de Bralten et al., cuyo título reza Los trastornos del espectro autista y los rasgos autistas comparten genética y biología ${ }^{(27)}$. De un modo u otro, los rasgos autistas se han constituido como un objeto de estudio en sí mismo, a tal punto que durante los últimos quince años se produjo un marcado incremento de publicaciones que buscan vincular la presencia de rasgos autistas con diversas condiciones o características particulares de individuos agrupados bajo diferentes criterios relacionados a la edad, género, demografía, presencia de patologías físicas o mentales previas, entre otros. Así, hay investigaciones que afirman la presencia elevada de rasgos autistas entre personas que han intentado cometer suicidio ${ }^{(9)}$, entre mujeres con anorexia nerviosa ${ }^{(10)}$, entre criminales de una prisión de máxima seguridad en Portugal ${ }^{(11)}$ entre personas en situación de indigencia ${ }^{(28)}$, entre nietas de abuelas maternas fumadoras $^{(29)}$, entre hombres con desorden de abuso de sustancias ${ }^{(30)}$, entre personas transgénero y de género diverso ${ }^{(31)}$, entre adultos jóvenes que nacieron en un parto extremadamente prematuro $^{(32)}$, y un largo etcétera.

Una amplia mayoría de estos trabajos implementa instrumentos denominados tests de screening, como la escala de capacidad de respuesta social $(S R S)^{(12)}$ o el cociente del espectro autista $(\mathrm{AQ})^{(33)}$, para la medición cuantitativa de rasgos autistas. Consisten en formularios cuyos ítems describen diversas conductas, algunas de ellas asociadas o semejantes al comportamiento de personas diagnosticadas con autismo como, por ejemplo, desarrollar una fascinación por los números, tener dificultades para hacer nuevas amistades, preferir ir a una biblioteca en lugar de una fiesta, no saber cómo detectar si la persona interlocutora se está aburriendo en una charla, etc. ${ }^{(33)}$. La metodología consiste en señalar aquellas afirmaciones con las que las personas evaluadas se identifican (o son identificadas, ya que los tests se llevan a cabo a modo de autoevaluación o, dependiendo del formato, el instrumento puede involucrar la participación de cuidadores de las personas evaluadas, sus docentes, profesionales de la salud, etc.), obteniendo un puntaje directamente proporcional a la cantidad de características asociadas al autismo seleccionadas y siendo los puntajes elevados indicadores de la presencia de rasgos autistas. Por eso, cuando se afirma que una característica dada se asocia a la manifestación de rasgos autistas, simplemente se establece una 
correlación estadística entre dicha característica y un puntaje alto de un determinado test de screening. Es sobre esta base que dos de las referentes más influyentes en la neurocognición del autismo afirman que

...las medidas de los rasgos autistas mediante el test AQ muestran un continuo fluido entre el autismo diagnosticado y las diferencias individuales subclínicas; [...] parece que, al menos a nivel conductual, uno puede ser "un poco autista". ${ }^{(14)}$

Pero, además, como Zhao et al. también afirman: "...se ha reconocido, desde hace mucho tiempo, que la gravedad de los síntomas del TEA se encuentra dentro de un espectro"(13). En efecto, desde que, en la década de 1970, Wing y Gould ${ }^{(34)}$ introdujeran la analogía entre la heterogeneidad de las manifestaciones del autismo y el espectro lumínico, la teoría de que el autismo representa los extremos de la distribución estadísticamente normal en la población total de rasgos autistas ${ }^{(35)}$ ha ido cobrando legitimidad con el paso del tiempo, especialmente, a partir de que Constantino y Todd publicaran en el año 2003 que los rasgos autistas se pueden hallar entre la población general. Así, en la bibliografía neurocientífica, los síntomas o criterios diagnósticos del TEA se consideran características cuantitativas que se pueden manifestar en mayor o menor grado, lo que podría explicar por qué estos se mencionan, con tanta frecuencia, de manera intercambiable, por ejemplo: "medir cuantitativamente los rasgos autistas o síntomas era necesario para medir las diferencias dentro del espectro"(14).

Resulta Ilamativo reconocer cómo se aborda el estudio de los rasgos autistas sin especificar una definición única y sin precisar cuáles son exactamente cada uno de ellos. Dada la linealidad con que se sostiene que las personas tienen elevados rasgos autistas si obtienen puntajes elevados en los tests de screening, se podría considerar que los rasgos autistas corresponden a los comportamientos descritos en los ítems en dichos instrumentos. No obstante, unos pocos trabajos remiten a los aspectos conductuales postulados por Dawson et al. ${ }^{(25)}$, mientras que una parte significativa los identifica con los síntomas. A esta indeterminación se añaden incongruencias marcadas por algunos detractores del uso de la noción de rasgos autistas. Por ejemplo, los investigadores Mottron y Bzdok $^{(36)}$ sostienen que las prácticas de diagnóstico actuales fragmentan el fenotipo del autismo en "rasgos autistas" lo que da como resultado el supuesto reconocimiento de síntomas asociados al autismo en cualquier condición psiquiátrica o del neurodesarrollo y en individuos cada vez menos distantes de la población no diagnosticada con enfermedades mentales. Esto conduce a un sobrediagnóstico de autismo, generando una falta de homogeneidad del fenotipo autista en las investigaciones que habría impedido a las neurociencias lograr mayores avances. Asimismo, sostienen que a la fecha no se ha demostrado la existencia de mecanismos biológicos que den origen o que se encuentren exclusiva y directamente vinculados a los rasgos autistas ${ }^{(36)}$.

\section{ENFOQUES FILOSÓFICOS SUBYACENTES A LOS RASGOS AUTISTAS}

A continuación, indagaremos acerca de los aspectos filosóficos y epistemológicos que subyacen a dichas representaciones neurocientíficas del autismo y buscaremos develar cuáles de ellos habilitaron el surgimiento, validación y consolidación de la distinción de rasgos autistas como manifestación subclínica de autismo.

\section{Concepción naturalista de las neurociencias}

Las neurociencias definen al autismo como una enfermedad del cerebro con fuertes bases genéticas, que deriva en alteraciones biológicas (bioquímicas, anatómicas, fisiológicas) y cognitivas, subyacentes a diversos 
comportamientos anormales a partir de los cuales se realiza el diagnóstico ${ }^{(1,14)}$. Dicha manera de caracterizar esta condición se corresponde con la visión naturalista de quienes sostienen que la dicotomía salud/enfermedad debe ser explicada a partir de definiciones objetivas y libres de valores, tomando el concepto de función natural como garantía de dicha objetividad. En 1997, Boorse introdujo la conceptualización bioestadística y fragmentada de salud sobre la que se asientan actualmente las teorías dominantes de las ciencias de la vida, considerando que cada segmento del organismo tiene una función que es natural y normal, en tanto se manifiesta de manera similar en la mayoría de los individuos de una población. Consecuentemente, la desviación estadística del funcionamiento de una determinada parte del cuerpo convierte a un individuo en enfermo; la enfermedad no es más que una disfunción biológica que se produce al interior del individuo $^{(37)}$. Desde este enfoque, las neurociencias, por un lado, conciben al autismo como un trastorno que se desencadena y transcurre al interior del individuo como consecuencia de disfunciones cerebrales (provocadas por diferentes alteraciones biológicas de causa predominantemente genética) y, por el otro, reproducen la definición bioestadística de la salud, al afirmar que el autismo no es más que los extremos de la distribución estadísticamente normal de rasgos autistas en toda la población. Ahora bien, este proceso a través del cual un trastorno mental se reduce en última instancia a una enfermedad física ocurre en sintonía con la pretensión positivista de procurar que los fenómenos se puedan medir objetivamente ${ }^{(38)}$, lo cual induce la suposición de que el mero incremento del conocimiento biológico permitirá y bastará para, eventualmente, alcanzar la cura de estas patologías ${ }^{(1)}$.

Aunque con menos adhesión en las neurociencias, otra aproximación posible es la normativista, que niega la idea de que la enfermedad pueda ser definida objetiva y científicamente como una desviación de una norma estadística, porque la caracterización de cualquier condición como desviada varía en relación con el trasfondo sociocultural, histórico y epistemológico. Oponiéndose a las concepciones deterministas y reduccionistas en salud, desde el normativismo se afirma que el significado que se otorga a las cosas posee un carácter subjetivo e intencional innegable y se cuestiona, en cambio, qué valores están en juego en la distinción entre lo normal y lo patológico ${ }^{(37)}$.

Debido a que estos posicionamientos -sobre todo en sus versiones más radicalizadas- traen aparejadas serias dificultades, como la exacerbación de un fuerte determinismo biológico en un caso y de un relativismo social extremo en el otro, numerosos autores han recurrido a los trabajos de Georges Canguilhem para elaborar propuestas tendientes a superar esta dicotomía entre naturalismo y normativismo, ya que este autor se opone a la concepción positivista de la salud desde el realismo biológico ${ }^{(18)}$.

\section{Aportes desde la epistemología canguilhemiana}

Para continuar con el análisis de los aspectos filosóficos, haremos un breve recorrido por algunos aspectos del pensamiento de Georges Canguilhem en su ensayo Lo normal y lo patológico. Dado que la obra está dedicada fundamentalmente al tratamiento de la fisiología y la medicina, también recuperamos reflexiones de Victoria Margree formuladas en su trabajo Normal y anormal: Georges Canguilhem y la pregunta de la patología mental ${ }^{(4)}$.

\section{La salud es relacional, dinámica e irreductible}

En 1943, Canguilhem define la vida como una actividad polarizada (o polaridad dinámica) y normativa, porque el organismo vivo se encuentra inmerso en un entorno que fluctúa permanentemente, al que evalúa de manera espontánea reaccionando negativa o positivamente según los estímulos resulten adversos o favorables para su supervivencia. El individuo regula esta interacción valorativa 
con su contexto mediante la modificación de sus patrones de comportamiento, que Canguilhem denomina como normas de vida. Introduce así la normatividad vital (o individual) que refiere a la capacidad que tienen los organismos de ajustarse a las circunstancias y que implica no solo seguir reglas, sino también ser capaz de instituir nuevas normas para uno mismo ${ }^{(4)}$; siendo además inherente a cada individuo como un todo, porque es el organismo en su "totalidad indivisible" que evalúa su entorno y reacciona ante sus cambios $^{(39)}$. Por lo tanto, la salud es entendida por Canguilhem como la capacidad plena de adaptación e invención de normas ante las variaciones del medio y como un estado creativo, propulsor y dinámico: "la salud es una forma de abordar la existencia, ya que uno siente que no solo es poseedor o portador, sino también, si es necesario, creador de valor, instalador de normas vitales"(39). Así, Margree apunta que, si la existencia sana es aquella que expresa más plenamente la normatividad vital, las normas saludables se caracterizan por su capacidad de autotrascendencia, siendo siempre provisionales y transitorias ${ }^{(4)}$.

Para Canguilhem, "decir que la salud perfecta no existe, significa solo decir que el concepto de salud no es el de una existencia, sino el de una norma cuya función y valor consisten en ser puesta en relación con la existencia para suscitar su modificación"(39). La salud no aparece como un objeto de estudio del que se pueden deducir las condiciones necesarias y suficientes para todos los estados sanos ${ }^{(4)}$. Por un lado, porque la salud es de naturaleza necesariamente indeterminada y dinámica, siendo incontenible dentro de parámetros fijos tales como aquellos que los naturalistas se empeñan en postular para poder realizar mediciones objetivas ${ }^{(4)}$. Y, por el otro, porque si el organismo se adapta como un todo indivisible a las fluctuaciones del entorno mediante su normatividad vital, la salud no puede ser fragmentada y reducida al funcionamiento de sus partes aisladas.

\section{El estado patológico representa una normatividad vital reducida}

Canguilhem argumenta que un organismo se halla en estado patológico cuando habita un "estrecho margen de tolerancia" hacia las variaciones de su entorno ${ }^{(39)}$. Lo patológico constituye una restricción de la normatividad individual porque la capacidad de adaptación e invención de nuevas normas se encuentra reducida y, en oposición a la salud, puede ser considerado como una fuerza conservadora. No obstante, como apunta Margree, si "anormal" se entiende literalmente "sin normas", los estados patológicos no son anormales: "lo anormal no es tal por ausencia de normalidad. No hay ningún tipo de vida sin normas de vida, y el estado mórbido es siempre una cierta manera de vivir"(39). De hecho, el estado patológico sigue operando de acuerdo con normas que, aunque se caracterizan por un menor potencial para afrontar el cambio, deben reconocerse como nuevas y originales ya que "la enfermedad es al mismo tiempo privación y remodelación"(39). Así, a diferencia de quienes afirman que el estado patológico difiere del sano de manera cuantitativa (por la disfunción de procesos normales en términos de excesos o deficiencias), Canguilhem sostiene que las normas patológicas representan una relación cualitativamente diferente entre el organismo y las circunstancias en términos de su capacidad para subsistir y desarrollarse ${ }^{(4)}$.

Al destacar numerosos casos médicos de estructuras o procesos estadísticamente anormales que no impiden la adaptación de un organismo a su medio ni resultan en la experiencia del sufrimiento, Canguilhem demuestra que las desviaciones cuantitativas de las normas promedio no son intrínsecamente patológicas y rechaza la asimilación de la patología a la anormalidad biológica en términos estadísticos porque lo patológico no puede determinarse únicamente a través de la medición ${ }^{(4)}$. Finalmente, devela cómo la pretensión de fijar parámetros de normalidad o de otorgar a un fenómeno particular la condición de patológico nunca es una operación objetiva en términos de que, se reconozca o no, 
implica llevar a cabo una acción valorativa ineludible: "esas categorías son biológicamente técnicas y subjetivas y no biológicamente científicas y objetivas. Los seres vivos prefieren la salud a la enfermedad"(39).

\section{El individuo no puede ser escindido de su entorno}

Canguilhem postula que como la vida es aqueIlo que se regula en función de su entorno, "el ser vivo y el medio ambiente no son normales tomados por separado, sino que su relación es la que los hace tales, tanto al uno como al otro" ${ }^{\prime(39)}$. Si la salud es relacional, en virtud de las interacciones entre individuo y contexto, las características biológicas no pueden considerarse patológicas de forma aislada: "no hay perturbación patológica en sí, lo anormal sólo puede ser apreciado dentro de una relación" ${ }^{\prime \prime 39)}$. Además, ningún hecho de la biología es patológico en un sentido absoluto porque una misma anomalía puede resultar restrictiva y desadaptativa o bien propulsora y creativa de acuerdo con los potenciales y limitaciones del contexto ${ }^{(4)}$. Consecuentemente, si el valor de una característica de un organismo varía según sus circunstancias, el objetivo de establecer determinados niveles de funciones como definitivamente patológicos se vuelve inalcanzable; para Canguilhem, "la persona enferma siempre debe ser juzgada en términos de la situación a la que está reaccionando y los instrumentos de acción que el propio entorno le ofrece" ${ }^{\prime \prime(39)}$.

Sin embargo, en el desarrollo de Canguilhem aparecen al menos dos acepciones con relación al entorno: una que refiere al medio interno del organismo y las interrelaciones entre sus componentes biológicas, y otra vinculada al ambiente exterior en el que se encuentra inmerso. Respecto de este último, particularmente el entorno humano involucra fenómenos de índole no solo vital sino social: "El hombre es un agente geográfico y la geografía está profundamente penetrada por la historia en forma de tecnologías colectivas"(39). Dada la interacción permanente entre el individuo humano y el contexto que le rodea, "en la especie humana la frecuencia estadística no sólo traduce una normatividad vital sino también una normatividad social"(39). Así, hechos en apariencia estrictamente biológicos son en realidad inseparablemente biológicos y sociales como "la duración promedio de vida [que] no es la duración de vida biológicamente normal, sino que en cierto sentido es la duración de vida socialmente normativa"(39). Por ello, "si se admite una plasticidad funcional del hombre, vinculada en él con la normatividad vital, no se trata de una maleabilidad total e instantánea, puramente individual"(39) sino que también estará afectada y limitada por la normatividad social.

\section{El enfoque neurocientífico desde la perspectiva de Canguilhem}

Uno de los interrogantes centrales de Lo normal y lo patológico es mediante qué estrategias las ciencias de la salud llevan a cabo el proceso de transformar datos estadísticos y descriptivos en ideales biológicos que determinan la normalidad y la salud. En el contexto del autismo, cabe preguntarse cómo las neurociencias otorgan una cualidad (cuasi)patológica a una noción descriptiva como la de los rasgos autistas. A partir del recorrido de la epistemología de Canguilhem, se reconocen tres supuestos que legitiman la consolidación de los rasgos autistas como manifestación del autismo subclínico. En primer lugar, al afirmar que los rasgos autistas se expresan como producto de disfunciones cerebrales, déficits neurocognitivos $y / o$ disturbios genéticos, se asume que los comportamientos humanos pueden y deben ser explicados estrictamente en términos biológicos. De este modo, la salud del individuo se reduce a los diferentes fragmentos de su organismo, cuya medición de manera aislada se espera que permita una determinación objetiva del fenómeno patológico, ignorando u omitiendo el valor que adquieren dichas "alteraciones" para el organismo y su potencialidad como un todo indivisible para adaptarse e instituir nuevas normas. En segundo lugar, cuando se describe al autismo como los extremos de la distribución estadísticamente normal de los 
rasgos autistas, se presupone que efectivamente existen parámetros de normalidad de carácter estadístico, objetivos y universales que al no ser alcanzados o al ser superados determinan la presencia del trastorno. Desde la óptica de Canguilhem, lo patológico no se puede asimilar a la desviación estadística porque entonces se niega que las anomalías pueden resultar adversas o favorables en relación con su entorno. Asimismo, la pretensión de establecer parámetros fijos y objetivos responde a un paradigma de salud estático, al que Canguilhem se opone por considerar las normas sanas como dinámicas en términos de ser provisionales y transitorias, y porque ningún hecho de la biología es patológico en un sentido absoluto. Por último, quienes Ilevan a cabo las mediciones de los rasgos autistas y quienes refieren a estas publicaciones como evidencia de la presencia de los rasgos autistas en las diferentes poblaciones analizadas, al utilizar instrumentos estandarizados para unas condiciones dadas asumen que los comportamientos de los individuos se manifiestan independientemente de su contexto. Como señalamos anteriormente, desde la perspectiva neurocientífica se asume que la patología se desencadena y transcurre al interior del organismo, subestimando la interacción del individuo con su entorno. Para Canguilhem, en cambio, los hechos son solo patológicos en virtud de sus interacciones porque una misma anomalía puede resultar desadaptativa o propulsora de acuerdo con los potenciales y limitaciones del ambiente, y porque el individuo no puede ser escindido de su entorno al punto tal que las normas sociales se ven reflejadas en las normas individuales y viceversa.

\section{DISCUSIÓN EPISTÉMICA SOBRE LOS RASGOS AUTISTAS}

Atendiendo a los numerosos cuestionamientos señalados por autores al interior del campo $y$ ante las incongruencias vinculadas a su definición, aparece el interés de revisar la noción de los rasgos autistas. En pos de profundizar el debate, proponemos una discusión epistémica para reflexionar acerca de los motivos por los cuales dicha noción resulta problemática, bajo qué términos se continúa legitimando su uso en el ámbito de las neurociencias y cuáles son los aspectos negativos que emergen desde este posicionamiento. Identificamos ciertas dificultades asociadas a los supuestos subyacentes a los rasgos autistas, cuya asunción acrítica deriva en prácticas y postulados que involucran una fundamentación positivista y biologicista, una desvinculación del sujeto con su contexto y un parámetro de normalidad esencialista y valorativo, lo cual impide poner en discusión aspectos elementales asociados a la naturaleza del autismo como el rol del medio y la dinámica propia de la vida.

\section{Fundamentación esencialista y biologicista}

Mediante la definición de vida como polaridad dinámica, Canguilhem trasciende el reduccionismo mediante el cual se afirma que la vida no es más que una mera función del mundo material y, en consecuencia, debe explicarse simplemente y en última instancia a través de los conceptos existentes de la química y la física ${ }^{(4)}$. Este tipo de pensamiento subyace tanto a aquellos enfoques dentro de las neurociencias que consideran necesario localizar en el cerebro la etiología de la enfermedad $^{(14,40)}$ como también, a los abordajes que buscan vínculos entre rasgos autistas y características biológicas ${ }^{(27)}$ o las miradas críticas que apuntan la falta de ellos $^{(36)}$. Bajo esta visión centrada en una explicación fundamentalmente reduccionista, biologicista y positivista, a partir de las ideas de que lo patológico ocurre y transcurre al interior del individuo, que se puede reducir su experiencia a procesos biológicos y que estos se pueden medir de manera objetiva, se habilita la imposición de características que se asocian al autismo como parámetros rígidos que aseguran una supuesta evaluación libre de valores y que son aplicables de manera universal a todos los individuos independientemente de sus contextos. 
Puesto que Canguilhem pone el foco en la normatividad individual para distinguir un estado sano de uno patológico, encontramos dos lecturas posibles de sus postulados en torno a la reducción de la vida humana a procesos biológicos medibles y universalizables. Por un lado, se ha argumentado que, si autismo refiere a acciones inflexibles/repetitivas y a formas rígidas de comunicación e interacción social, este trastorno puede ser considerado como un claro ejemplo de enfermedad desde la perspectiva de Canguilhem ${ }^{(3)}$. Sin embargo, cuando afirma que "un individuo con autismo tiende a favorecer un entorno estable y predecible para minimizar la posibilidad de estrés y sufrimiento, y es incapaz de responder a los acontecimientos inesperados de forma creativa y flexible ${ }^{\prime \prime(4)} \mathrm{se}$ entiende que este autor está contemplando al sujeto en su totalidad a la vez que repara en su actividad normativa y en el valor que adquieren sus circunstancias, tal como sugiere Canguilhem. Por el contrario, los rasgos autistas representan desviaciones estadísticas, se formulan como un listado de comportamientos escindidos de los individuos, se evalúan aislados del contexto y se busca vincularlos con determinadas alteraciones biológicas y cognitivas como garantía de veracidad. Recuperando las ideas de Canguilhem, se podría pensar que estos comportamientos anormales en realidad constituyen normas adaptativas que surgen como producto de un entorno desfavorable para el desenvolvimiento de los individuos. De esta manera, si tenemos en cuenta que una misma anomalía puede resultar restrictiva o propulsora en función de los potenciales y limitaciones de cada individuo en relación con su entorno, sería erróneo considerar que los rasgos autistas descritos como síntomas del DSM (o ítems de los tests de screening) son (cuasi)patológicos a priori, así como también que son patológicos para todos los individuos por igual, es decir, generalizables a toda la población omitiendo la dimensión social e histórica de cada individuo. En palabras de Caponi para el caso de la depresión: ...los criterios diagnósticos [...] se refieren a conductas genéricas de sujetos indefinidos: viejos o jóvenes, pobres o ricos, desempleados o empresarios. Esto es, las conductas son evaluadas ignorando las demandas que, en cada caso concreto, el medio impone a cada sujeto. ${ }^{(17)}$

Además, Buchanan señala que cuando los manuales psiquiátricos incorporan la noción de adaptación:

...por lo general, se hace hincapié en la capacidad de una persona para funcionar en las circunstancias en las que se encuentra $y$, con menos frecuencia, en su historial de ser capaz de responder al cambio, de funcionar eficazmente en una variedad de roles y de continuar haciéndolo cuando las circunstancias requieren que esos roles cambien. ${ }^{(19)}$

\section{Parámetro de normalidad basado en comportamientos socialmente aceptados}

La configuración del autismo como los extremos de una distribución normal en términos estadísticos de rasgos autistas se encuentra consolidada en el ámbito de las neurociencias porque posibilita determinar la enfermedad únicamente mediante una medición objetiva de una desviación cuantitativa de una norma, a priori considerada como la única manera de abordar y examinar los estados anormales y patológicos. Sin embargo, como apunta Canguilhem:

\footnotetext{
...exceso y defecto existen con respecto a una medida que se considera válida y deseable, por lo tanto, con respecto a una norma. Definir lo anormal por lo demasiado o por lo demasiado poco, significa reconocer el carácter normativo del denominado "estado normal". (39)
}

Es decir que detrás de estos parámetros cuantitativos subyacen aspectos implícitamente evaluativos y normativos que el naturalismo 
niega con su pretendida neutralidad. Para el caso del autismo, es imposible omitir el profundo componente social que lo caracteriza, en tanto un grupo de síntomas o rasgos autistas que se describen como dificultades en la interacción social y la comunicación. Cabe la sospecha entonces de que existan normas sociales vinculadas a comportamientos deseables ${ }^{(17)}$, aceptados y permitidos, que se reflejen en el umbral respecto del cual un carácter resulta excesivo o deficiente, de modo tal que si alguien escapa a esa norma es considerado a priori como patológico. En este sentido, Canguilhem considera una equivocación identificar a las características exitosas consideradas normales con las características promedio debido a que "un rasgo humano no sería normal porque fuese frecuente, sino a la inversa: sería frecuente por ser normal, es decir normativo en un género de vida dado $^{\prime \prime(39)}$. Si bien refiere con esto a características más bien de tipo biológicas como la altura o la longevidad, podríamos extender esta afirmación al hecho de que las normas socialmente aceptadas quizá son estadísticamente promedio porque se margina, se descarta o se busca normalizar a quienes habitan normas socialmente inaceptables. De hecho, para Canguilhem "normalizar significa imponer una exigencia a una existencia, a un dato, cuya variedad y disparidad se ofrecen, con respecto a la exigencia, más aún como algo indeterminado y hostil que simplemente como algo extraño" ${ }^{\prime(39)}$.

Desde esta perspectiva, el criterio para calificar cualquier hecho comportamental o psicológico como patológico no debería ser entonces su desviación estadística, sino su efecto en términos de la restricción de la normatividad individual que sea percibida como una experiencia limitante y de sufrimiento. Así,

...si lo normal no tiene la rigidez de un hecho de obligación colectiva sino la flexibilidad de una norma que se transforma en su relación con condiciones individuales, es evidente que la frontera entre lo normal y lo patológico se hace imprecisa. [Sin embargo] es imprecisa para los múltiples individuos considerados simultáneamente, pero es perfectamente precisa para un solo e idéntico individuo considerado sucesivamente. ${ }^{(39)}$

En consecuencia, como apunta Hens, es importante incorporar la experiencia vivida de las personas autistas en la investigación ${ }^{(20)}$.

\section{Desvinculación del sujeto con su contexto y subestimación del rol del entorno}

Para ser cuantificados de manera objetiva, los rasgos autistas deben ser genéricos y referirse a sujetos indefinidos y sin entorno. Además, en pos de asegurar esta presunta imparcialidad, las evaluaciones se realizan generalmente en "condiciones controladas" como laboratorios o salas especiales, porque se asume que así se evitan estímulos externos que afecten las mediciones. Canguilhem señala que, aún así, estos entornos constituyen contextos ajenos a la cotidianeidad de los individuos, lo cual no solo tiene como consecuencia la imposibilidad de analizar la relación entre el individuo y su entorno habitual, sino que en el marco de un contexto novedoso se incrementa la probabilidad de que el organismo genere nuevas normas en ese momento para adaptarse a un contexto extraño ${ }^{(7)}$. No obstante, más allá de su cuantificación, la propia formulación de los rasgos autistas excluye a priori el rol del ambiente, o en su defecto lo relega a un papel secundario. Si bien han aparecido abordajes vinculados a la epigenética, estos afirman que los factores ambientales son meros modificadores genéticos, expresando además un determinismo genético fuertemente arraigado en un marco naturalista que pondera a los genes como causas principales de las patologías ${ }^{(2)}$.

En contraste, Canguilhem remarca la imposibilidad de negar el rol del entorno como un componente esencial de la vida y afirma que tanto el individuo como su contexto son producto al mismo tiempo de la actividad normativa individual y de la normatividad social. De este modo, "queda en evidencia 
que no es el simple reconocimiento de la interacción individuo-medio, sino también el modo como se establece esa relación lo que debe ser considerado cuando hablamos de salud"(15). Aparecen dimensiones de carácter social, ético-político, económico, geográfico, histórico, tecnológico, etc., que las neurociencias no suelen contemplar y que la fundamentación naturalista impide incorporar por considerar que la enfermedad se origina y transcurre solamente al interior de los individuos. En este sentido, tanto el uso de pesticidas como el caso de "los huérfanos rumanos"(43) permiten ilustrar la relevancia que tienen dichos aspectos en el caso del autismo. Por un lado, hay trabajos que afirman haber encontrado altas tasas de autismo entre las poblaciones aledañas a las zonas donde se utilizan pesticidas como el glifosato ${ }^{(41)}$. Por el otro, existen personas cuyo comportamiento recibió la controversial denominación de "autismo institucionalizado" ${ }^{(42)}$ y se produjo como consecuencia de la negligencia del Estado rumano en el cuidado (en términos de la deprivación de alimentación, aseo y afecto) de niñxs huérfanxs durante las décadas del 1960 y $1970^{(43)}$. Estos ejemplos muestran cómo ciertas intervenciones tecnológicas en el ambiente y determinadas políticas públicas pueden propiciar condiciones biológicas y psicológicas perjudiciales, en términos de entornos adversos que restringen la normatividad individual y por la cual emerge un estado patológico causado y dependiente del contexto.

\section{CONCLUSIÓN}

En este trabajo partimos de que la experiencia del autismo puede constituirse como un fenómeno patológico y puede provocar sufrimiento a quien lo experimenta. Lejos de considerar que es una categoría ficcional, abonamos a la idea de que no se puede banalizar la terminología ni generalizar sus características haciéndolas extensivas a cualquier individuo de manera apriorística. Por lo tanto, el interés que moviliza el presente trabajo surge a raíz de la proliferación de publicaciones en el ámbito de las neurociencias, que indican la presencia de rasgos autistas en diferentes grupos de individuos y posibilita que sobrevuele la idea de que cualquiera puede ser susceptible de ser considerado "un poco autista".

Aunque existen aproximaciones que intentan contemplar al individuo como un todo $^{(44)}$, que incorporan el análisis del contexto $^{(45)}$ o que abogan por la flexibilidad de los parámetros de normalidad ${ }^{(46)}$, observamos que generalmente en la bibliografía neurocientífica este concepto se valida y se reproduce de manera acrítica en el marco de una perspectiva naturalista de la salud y la enfermedad asumida como la única visión posible, a través de la consolidación de una serie de presupuestos que se internalizan en la práctica científica sin mediar problematización alguna. Hallamos concepciones biologicistas, deterministas y positivistas fuertemente arraigadas en la pretensión de formular parámetros genéricos, fijos y medibles que se puedan universalizar a toda la población omitiendo el papel crucial del entorno.

Como consecuencia, nos preguntamos si acaso esta práctica promueve una medicalización del comportamiento humano al reducir la experiencia patológica que constituye el autismo a una serie de rasgos con los que muchas personas no autistas pueden identificarse fácilmente. Consideramos que los aportes de Canguilhem permiten superar estas dificultades porque entiende que lo que determina los estados patológicos no son simplemente las nociones métricas, sino el valor que adquieren las características individuales dependiendo de las circunstancias. Además, si las mismas características pueden resultar patológicas en algunas condiciones y saludables en otras, la enfermedad no se ubica simplemente dentro del organismo, sino que reside en las relaciones recíprocas con su entorno. También demuestra que los estándares humanos se presentan como hechos dados cuando en verdad son parámetros construidos socialmente y evaluados psicológicamente. De este modo, lo normal y lo patológico varía según los individuos, pero 
también según los momentos y contextos. Esta perspectiva que pone en valor la experiencia individual y contempla al individuo en su totalidad, involucra necesaria e inseparablemente lo biológico, lo psicológico y lo social $^{(47)}$.

Por otro lado, entender a las patologías simplemente como una desviación cuantitativa de un conjunto de constantes, supone que se puede convertir lo patológico nuevamente en lo normal a través de la intervención humana experta en las funciones normales. Como destaca Lefève ${ }^{(47)}$, Canguilhem se opone tanto a la concepción individualista como a la concepción colectivista que se puede desprender de esta visión. En primer lugar, porque la concepción individualista implica considerar que la enfermedad se ubica al interior del individuo, cuyos estándares se construyen sin el impacto de las normas sociales. En segundo lugar, porque la concepción colectivista habilita que, como es el caso de comportamientos asociados al autismo, la búsqueda de restituir la salud resulte equivalente a realizar una normalización según estándares sociales. Esta doble oposición es clave porque cualquiera de ellas libera a la sociedad de la responsabilidad de transformarse y adaptarse a la individualidad en general y al paciente en particular ${ }^{(47)}$.

Para el ámbito de las neurociencias podría resultar beneficioso poner en discusión aspectos elementales asociados a la naturaleza del autismo como el rol del medio y la dinámica propia de la vida. Mediante la reducción de la experiencia autista a un conjunto de constantes biológicas, parámetros conductuales y patrones comportamentales e ignorando las vivencias y circunstancias individuales "nos reservamos la posibilidad de que la vida vaya más allá de las constantes o invariantes biológicas codificadas que se sostienen convencionalmente como normas en un momento específico del conocimiento"(39). Pero, fundamentalmente, es momento de reflexionar acerca del impacto que tiene la actividad científica y evitar prácticas tendientes a la patologización de la vida y las diferencias humanas, como la formulación de nuevas distinciones y categorías que se terminan asumiendo apriorísticamente como equivalentes a expresiones patológicas.

\section{FINANCIAMIENTO}

Este artículo es un producto parcial del proyecto "Análisis epistemológico comparativo sobre las conceptualizaciones del autismo propuestas por las perspectivas neurocientífica y psiquiátrica", realizado en el marco de la beca de doctorado 2018-2023 otorgada a Clara Castañares por el Consejo Nacional de Investigaciones Científicas y Técnicas (CONICET).

\section{AGRADECIMIENTOS}

Agradecemos al Grupo de Filosofía de la Biología por su compromiso y apoyo durante el desarrollo de este trabajo.

\section{CONFLICTO DE INTERESES}

Las autoras declaran no tener vínculos o compromisos que condicionen lo expresado en el texto y que puedan ser entendidos como conflicto de intereses.

\section{REFERENCIAS BIBLIOGRÁFICAS}

1. Verhoeff B. Autism's anatomy: A dissection of the structure and development of a psychiatric concept. Groningen: University of Groningen; 2015.

2. Kendler K, Parnas J. Philosophical issues in psychiatry II: Nosology. Oxford: Oxford University Press; 2017.

3. Verhoeff B. Normaal of pathologisch? - De visie van arts en filosoof Georges Canguilhem (1904-1995). Tijdschrift Voor Psychiatrie. 2010;52(9):639-647.

4. Margree V. Normal and abnormal: Georges Canguilhem and the question of mental pathology. Philosophy, Psychiatry, \& Psychology. 2002;9(4):299-312.

5. Ferreyra JA, Castorina JA. El aplicacionismo de las neurociencias en el campo de la salud mental. Investigaciones en Psicología. 2017;22(2):25-36. 
6. Bloise L, Arias C, Folguera G. Problematizando a la neurociencia cognitiva: biologicismo, patologización y narrativas para el control social. I Jornadas de Fundamentos y Aplicaciones de la Interdisciplina [Internet] 2021 [citado 12 nov 2021]. Disponible en: https:// tinyurl.com/2p83jxwm.

7. Lavagnino NJ, Barbero S, Folguera G. Caracterización, alcances y dificultades de las "bases biológicas" del Trastorno por Déficit de Atención e Hiperactividad (TDAH). Un enfoque desde la Filosofía de la Biología. Physis: Revista de Saúde Coletiva. 2018;28(1):1-32.

8. Bracken $\mathrm{P}$, Thomas $\mathrm{P}$, Timimi $\mathrm{S}$, Asen $\mathrm{E}$, Behr $\mathrm{G}$ Beuster C, et al. Psychiatry beyond the current paradigm. British Journal of Psychiatry. 2012;201(6):430-434.

9. Richards G, Kenny R, Griffiths S, Allison C, Mosse D, Holt R, et al. Autistic traits in adults who have attempted suicide. Molecular Autism. 2019;10(1):4-13.

10. Fornaro $M$, Sassi $T$, Novello $S$, Anastasia A, Fusco A, Senatore I, et al. Prominent autistic traits and subthreshold bipolar/mixed features of depression in severe anorexia nervosa. Brazilian Journal of Psychiatry. 2020;42(2):153-161.

11. Loureiro D, Machado A, Silva T, Veigas T, Ramalheira $C$, Cerejeira J. Higher autistic traits among criminals, but no link to psychopathy: Findings from a high-security prison in Portugal. Journal of Autism and Developmental Disorders. 2018;48(9):3010-3020.

12. Constantino JN, Todd RD. Autistic traits in the general population: A twin study. Archives of General Psychiatry. 2003;60(5):524-530.

13. Zhao X, Shi W, Li X, Li W, Li C. Autistic traits and negative emotions in the general population during COVID-19: Mediating roles of the behavioural immune system and COVID-19 risk perception. Psychiatry Research. 2021:300:113918.

14. Happé F, Frith U. Annual Research Review: Looking back to look forward - changes in the concept of autism and implications for future research. Journal of Child Psycholofy and Psychiatry. 2020;61(3):218-232.

15. Caponi S. Georges Canguilhem: del cuerpo subjetivo a la localización cerebral. Salud Colectiva. 2010;6(2):149161.

16. Neves TI, Porcaro LA, Curvo DR. Saúde é colocar-se em risco: normatividade vital em Georges Canguilhem. Saude e Sociedade. 2017;26(3):626-637.

17. Caponi S. An epistemological analysis of the diagnosis of depression. Interface - Comunicação Saúde Educação. 2009;13(29):327-338.

18. Greco M. Between social and organic norms: reading Canguilhem and 'somatization'. Economy and Society. 1998;27(2-3):234-248.

19. Buchanan A. Georges Canguilhem and the diagnosis of personality disorder. The Journal of the American Academy of Psychiatry and the Law. 2007;35(2):148-151.
20. Hens K. The many meanings of autism: conceptual and ethical reflections. Developmental Medicine \& Child Neurology. 2019;61(9):1025-1029.

21. Lord C, Brugha TS, Charman T, Cusack J, Dumas G, Frazier T, et al. Autism spectrum disorder. Nature Reviews Disease Primers. 2020;6(1):5.

22. Nadesan $\mathbf{M H}$. Constructing Autism: Unravelling the 'truth' and understanding the social. Constructing Autism. New York: Routledge; 2005.

23. American Psychiatric Association. Diagnostic and Statistical Manual of Mental Disorders. 5th ed. Washington: American Psychiatric Association; 2013.

24. Chown N. Are the "autistic traits" and "broader autism phenotype" concepts real or mythical? Autism Policy and Practice. 2019;2(1):46-63.

25. Dawson G, Webb S, Schellenberg GD, Dager S, Friedman S, Aylward E, et al. Defining the broader phenotype of autism: Genetic, brain, and behavioral perspectives. Development and Psychopathology. 2002;14(3):581-611.

26. Maxwell CR, Parish-Morris J, Hsin O, Bush JC, Schultz RT. The broad autism phenotype predicts child functioning in autism spectrum disorders. Journal of Neurodevelopmental Disorders. 2013;5:25.

27. Bralten J, Van Hulzen KJ, Martens MB, Galesloot TE, Arias Vasquez A, Kiemeney LA, et al. Autism spectrum disorders and autistic traits share genetics and biology. Molecular Psychiatry. 2018;23(5):1205-1212.

28. Churchard A, Ryder M, Greenhill A, Mandy W. The prevalence of autistic traits in a homeless population. Autism. 2019;23(3):665-676.

29. Golding J, Ellis G, Gregory S, Birmingham K, llesCaven $\mathrm{Y}$, Rai D, et al. Grand-maternal smoking in pregnancy and grandchild's autistic traits and diagnosed autism. Scientific Reports. 2017;7:46179.

30. Işik Ü, Çokünlü $Y$, Şimşek F, Demirel B, Kiliç $F$, Demirdaş $A$, et al. Autistic traits in men with substance use disorder. The Journal of Nervous and Mental Disease. 2020;208(6):505-509.

31. Warrier V, Greenberg DM, Weir E, Buckingham C, Smith P, Lai MC, et al. Elevated rates of autism, other neurodevelopmental and psychiatric diagnoses, and autistic traits in transgender and gender-diverse individuals. Nature Communications. 2020;11:3959.

32. O'Reilly H, Ni Y, Johnson S, Wolke D, Marlow N. Extremely preterm birth and autistic traits in young adulthood: the EPICure study. Molecular Autism. 2021;12:30.

33. Baron-Cohen S, Hoekstra rasgos autistas, Knickmeyer R, Wheelwright S. The Autism-Spectrum Quotient (AQ)_Adolescent Version. Journal of Autism and Developmental Disorders. 2006;36(3):343-350.

34. Wing L, Gould J. Severe impairments of social interaction and associated abnormalities in children: epi- 
demiology and classification. Journal of Autism and Developmental Disorders. 1979;9(1):11-29.

35. Whitehouse AJ, Hickey M, Ronald A. Are autistic traits in the general population stable across development? PLoS One. 2011;6(8):e23029.

36. Mottron L, Bzdok D. Autism spectrum heterogeneity: fact or artifact? Molecular Psychiatry. 2020;25:3178-3185.

37. Gaudenzi P. Normal e patológico no naturalismo e no normativismo em saúde: a controvérsia entre Boorse e Nordenfelt. Physis: Revista de Saúde Coletiva. 2016;26(3):747-767.

38. Rose N. Neuroscience and the future for mental health? Epidemiology and Psychiatric Sciences. 2016;25(2):95100.

39. Canguilhem G. Lo normal y lo patológico. Buenos Aires: Siglo XXI Editores; 1971.

40. London E. The role of the neurobiologist in redefining the diagnosis of autism. Brain Pathology. 2007;17(4):408-411.

41. von Ehrenstein OS, Ling C, Cui X, Cockburn M, Park AS, Yu F, et al. Prenatal and infant exposure to ambient pesticides and autism spectrum disorder in children: po- pulation based case-control study. BMJ. 2019;364:1962.

42. Gindis B. Institutional autism in children adopted internationally: Myth or reality? International Journal of Special Education. 2008;23(3):118-123.

43. Rutter M, Andersen Wood L, Beckett C, Bredenkamp D, Castle J, Groothues C, et al. Quasi autistic patterns following severe early global privation. Journal of Child Psychology and Psychiatry, and Allied Disciplines. 1999;40(4):537-549.

44. Hull L, Lai MC, Baron-Cohen S, Allison C, Smith P, Petrides K V., et al. Gender differences in self-reported camouflaging in autistic and non-autistic adults. Autism. 2020;24(2):352-563.

45. de Leeuw A, Happé F, Hoekstra RA. A conceptual framework for understanding the cultural and contextual factors on Autism across the globe. Autism Research. 2020;13(7):1029-1050.

46. Happé F, Frith $U$. The beautiful otherness of the autistic mind. Philosophical Transactions of the Royal Society of London: Series B, Biological Sciences. 2009;364(1522):1345-1350.

47. Lefève C. La relation médecin-malade chez Georges Canguilhem. Dialogue. 2013;52(4):695-723.

\section{FORMA DE CITAR}

Castañares C, Bagatolli L, Folguera G. Análisis filosófico sobre la concepción neurocientífica de los rasgos autistas: aportes desde el normativismo vital de Georges Canguilhem. Salud Colectiva. 2021;17:e3809. doi: 10.18294/ sc. 2021.3809 .

Recibido: 13 sep 2021 | Versión final: 29 nov 2021 | Aprobado: 1 dic 2021 | Publicado en línea: 20 dic 2021

Esta obra está bajo una licencia Creative Commons Atribución 4.0 Internacional (CC BY 4.0). Atribución - Se debe dar crédito de manera adecuada, brindar un enlace a la licencia, e indicar si se han realizado cambios. Puede hacerlo en cualquier forma razonable, pero no de forma tal que sugiera que usted o su uso tienen el apoyo de la licenciante. Sin restricciones adicionales - No se pueden aplicar términos legales ni medidas tecnológicas que restrinjan legalmente a otras personas a hacer cualquier uso permitido por la licencia. 